\title{
Symmetry breakdown and coupling constants of leptons
}

\author{
GIL C. MARQUES ${ }^{1}$ and DOMINIQUE SPEHLER ${ }^{2}$ \\ ${ }^{1}$ Instituto de Física, Universidade de São Paulo, Caixa Postal 66318, 05315-970 São Paulo, SP, Brazil \\ ${ }^{2}$ IPHC-DRS, ULP, CNRS in2p3, 23 Rue du loess, 67037 Strasbourg, France \\ Manuscript received on September 22, 2006; accepted for publication on March 3, 2007; \\ contributed by GIL C. MARQUES*
}

\begin{abstract}
Based on a new approach to symmetries of the fundamental interactions we deal, in this paper, with the electroweak interactions of leptons. We show that the coupling constants, arising in the way leptons are coupled to intermediate bosons, can be understood as parameters associated to the breakdown of $S U$ (2) and parity symmetries. The breakdown of both symmetries is characterized by a new parameter (the asymetry parameter) of the electroweak interactions. This parameter gives a measure of the strength of breakdown of symmetries. We analyse the behaviour of the theory for three values of this parameter. The most relevant value is the one for which only the electromagnetic interactions do not break parity (the maximally allowed left-right asymetric theory). Maximamally allowed parity asymmetry is a requirement that is met for a value of Weinberg's $\theta$-angle that is quite close to the experimental value of this parameter.
\end{abstract}

Key words: symmetries, coupling constants, eletroweak interactions.

\section{INTRODUCTION}

Search of symmetry is a relevant tool in order to discover the laws of nature and the purist guiding principles to achieve a logical description of nature. Symmetry is probably the only thing one has do know besides quantum theory. Symmetries play an essential role in the model building process for understanding the properties of the Elementary Particles and their interactions. Differences in the electric charges of fermions and in the masses of the particles indicate that some symmetries are not exact. This makes the understanding of breakdown of symmetries an equally important issue in Particle Physics.

In Particle Physics we deal with a large number of symmetries: translational, rotational, Lorentz invariance, chiral symmetry, dual symmetry, $C$ (charge), $P$ (parity) and $T$ (time reversal) symmetries, gauge symmetry, and internal symmetries, like $S U(2)$ and $S U(3)$ group symmetries. Different interactions are (in the usual framework) governed by different symmetries [1-4].

\footnotetext{
* Member Academia Brasileira de Ciências

Correspondence to: Gil C. Marques

E-mail: marques@if.usp.br
} 
The Standard Model [1] makes use of two interactions exhibiting $S U(2)$ and $U(1)$ gauge symmetries. In the GWS theory these symmetries are broken trough the use of two mechanisms. The mixings of the electromagnetic and neutral weak interaction boson fields take into account differences in the interactions of leptons (including differences in their electric charges). Spontaneously symmetry breakdown is used as a mechanism for providing masses to particles.

Parity violation is taken into account, in the Standard Model, by assigning chiral components of fermionic fields to different representations of the $S U(2)$ group.

In this paper we propose a different view on the symmetries of the electromagnetic and weak interactions. We show that the underlying symmetry of the interactions of leptons is the $S U(2)$ symmetry. In our approach, there is only one type of interaction and only one coupling constant. We are proposing also different mechanisms for the breakdown of parity and $S U(2)$ symmetries.

Breakdown of symmetries introduces a free parameter in the theory (the assymetry parameter $-\xi$ ). The parameter $\xi$ gives a measure of breakdown of symmetries. As we shall see, the theory exhibits different types of symmetries (or asymmetries) for three different values of this parameter. For $\xi=1$ one gets a symmetry that is related to parity breakdown in the charged and neutral sector. For $\xi=0$ one gets an $S U$ (2) symmetric theory for which parity is completely broken. As we shall see later, the most relevant case is the one for which $\xi^{2}=1 / 3$.

The dynamical variables, used in the description of spin 1 particles, are chiral components of rank 2 spinor fields [5-11]. In our approach fermions belongs to doublet representations of the $S U$ (2) group. No use is made of fermionic field components transforming as singlets under $S U$ (2) transformations.

The use of spinor fields for the description of bosons and fermions allows us to formulate a model of the fundamental interactions based on space-time, discrete symmetries $(C P T)[8,9]$ and internal $S U(3) \times$ $S U(2)$ global symmetries $[10,11]$.

The plan of this paper is the following:

In Section 2 we present our dynamic variables by using rank 1 spinor fields (for fermions) and rank 2 spinor fields (for bosons). We discuss also how we deal with internal symmetries, like $S U(2)$ symmetry.

The most general theory involving the interaction of fermions is presented in Section 3.

The relevant aspect of breakdown of isospin and space-reflection symmetries, and our way of dealing with these questions is discussed in Section 4 . We introduce a parameter $-\xi$ - that establishes the strength of the breakdown of both symmetries. All coupling constants, electric charges included, can be expressed in terms of this parameter.

In Section 5 we present an application of our approach. We discuss how $S U(2)$ and parity symmetry breakdown gives rise to two different interactions and how these interaction strengths depend on the symmetry breakdown parameter. Electromagnetic interactions arises in the process of breaking $S U(2)$ symmetry. Electric charges of leptons depend on the asymmetry parameter in such a way that electric charges has to vanish in order to restore $S U(2)$ symmetry.

We end this paper with some conclusions in Section 6. 


\section{DYNAMICAL VARIABLES}

We take for grant that an unified theory can be formulated based on space-time, discrete and global internal symmetries [9-11]. This is so because we work, in our framework, with spinor fields. We would like to call the attention to the fact that depending on how we couple some components of rank-2 spinor fields one can end up with a theory that, besides being globally symmetric, is also locally symmetric. The general conditions under which this happens is, up to this point, not clear. One can give however, some explicit examples $[9,10]$. The point is that, by working with spinor fields we do not discard a gauge invariant theory.

In order to take into account the necessary Lorentz invariance of the theory we deal with two different types of fields.

Fermions are described by a doublet of rank 1 spinor fields. In this paper we will be concerned only with left-handed neutrinos. For left-handed particles it is convenient to define the $L^{(F)}$ (where F stands for the F-family) fields

$$
L_{i_{1}, a_{1}}^{(F)}=\mathcal{P}_{i_{1} i_{2}, a_{1} a_{2}} \ell_{i_{2}, a_{2}}^{(F)}
$$

where the matrix $\mathcal{P}_{i_{1} i_{2}, a_{1} a_{2}}$ in (2.1) is given by:

$$
\mathcal{P}_{i_{1} i_{2}, a_{1} a_{2}}=\left(\begin{array}{cc}
\frac{1}{2}\left(1-\gamma^{5}\right)_{a_{1} a_{2}} & 0 \\
0 & 1_{a_{1} a_{2}}
\end{array}\right) .
$$

The important difference in dealing with fermions, as we compare with the Standard Model, is that we do not use singlet representations for fermions.

For the description of the intermediate bosons one uses a rank 2 spinor field. Being a rank 2 spinor field it carries two spinor indices $\left(a_{1} a_{2}\right)$ running from 1 to 4 . This spinor field will be denoted by:

$$
\psi_{i_{1} i_{2}, a_{1} a_{2}}^{(2)}(x) \quad i_{1}, i_{2}=1,2,
$$

where the i-indices are internal symmetry indices. From (2.3) it follows that the fundamental spinors associated to bosons transform as a direct product of two fundamental representations of the Lorentz and $S U(2)$ groups.

The use of rank 2 spinor fields introduces, however, a large number of field components (in the Lorentz indices). This requires that, in dealing with spinor fields, we treat the chiral components of these fields as independent dynamical variables of the theory [6-10]. The chiral components of a rank 2 spinor field are defined, in close analogy with the way we define chiral components of rank 1 spinor fields, as:

$$
\begin{aligned}
& \psi_{i_{1} i_{2} R R}=\frac{1}{2}\left(1+\gamma^{5}\right) \otimes \frac{1}{2}\left(1+\gamma^{5}\right) \psi_{i_{1} i_{2}} \\
& \psi_{i_{1} i_{2} R L}=\frac{1}{2}\left(1+\gamma^{5}\right) \otimes \frac{1}{2}\left(1-\gamma^{5}\right) \psi_{i_{1} i_{2}} \\
& \psi_{i_{1} i_{2} L R}=\frac{1}{2}\left(1-\gamma^{5}\right) \otimes \frac{1}{2}\left(1+\gamma^{5}\right) \psi_{i_{1} i_{2}} \\
& \psi_{i_{1} i_{2} L L}=\frac{1}{2}\left(1-\gamma^{5}\right) \otimes \frac{1}{2}\left(1-\gamma^{5}\right) \psi_{i_{1} i_{2}} .
\end{aligned}
$$


Our Unified Model will be formulated for a generic family $F$ in terms of just two dynamical variables. We use one rank 1 spinor field for describing leptons and one rank 2 spinor field for the description of the intermediate bosons.

By definition, the bosonic field transforms as a direct product of fundamental representations of the groups. By using a basis of orthogonal matrices we can decompose any such a product as a sum over fields transforming as irreducible representations of the symmetry groups. This can be done if one decomposes the fields $\psi_{a_{1} a_{2}}$ into the basis formed by a convenient set of $k$ matrices $M_{a_{1} a_{2}}^{(k)}$. They are assumed to be traceless, orthogonal and linearly independent matrices. That is, one writes a decomposition under the form

$$
\psi_{a_{1} a_{2}}(x)=\sum_{k} M_{a_{1} a_{2}}^{(k)} \psi^{(k)}(x),
$$

in such a way that the $\psi^{(k)}$ components of the field $\psi$ transform (as we shall see later) as irreducible representations of the group.

Let us analyse first the Lorentz Group. In this case one needs a basis of 16 matrices. We take these matrices as the set of the 16 linearly independent $\gamma$-matrices [8]: $\gamma^{\mu} C, \gamma^{5} \gamma^{\mu} C, \sigma^{\mu \nu} C, C$ and $\gamma^{5} C$. We write:

$$
\begin{aligned}
\psi_{i_{1} i_{2}, a_{1} a_{2}}= & \left(\gamma^{\mu} C\right)_{a_{1} a_{2}}\left(G_{\mu}^{V}\right)_{i_{1} i_{2}}+\left(\gamma^{5} \gamma^{\mu} C\right)_{a_{1} a_{2}}\left(G_{\mu}^{A}\right)_{i_{1} i_{2}}+\left(\sigma^{\mu \nu} C\right)_{a_{1} a_{2}}\left(\mathcal{F}_{\mu \nu}\right)_{i_{1} i_{2}} \\
& +(C)_{a_{1} a_{2}}(\phi)_{i_{1} i_{2}}+\left(\gamma^{5} C\right)_{a_{1} a_{2}}\left(\phi^{A}\right)_{i_{1} i_{2}} .
\end{aligned}
$$

Expression (2.9) allows us to decompose a rank 2 spinor field in terms of an antisymmetric tensor field $\left(\mathcal{F}_{\mu \nu}\right)$, two rank 1 vector field $\left(G_{\mu}^{V}\right.$ and $\left.G_{\mu}^{A}\right)$ and two scalar fields $\left(\phi\right.$ and $\left.\phi^{A}\right)$.

In order to describe the weak interactions, we need both the vector $\left(G_{\mu}^{V}\right)_{i_{1} i_{2}}$ and the pseudovector $\left(G_{\mu}^{A}\right)_{i_{1} i_{2}}$ fields. For the $S U(3)$ group one needs to take only symmetric (in the spinor indices) rank 2 spinor fields, so that one needs only a vector $\left(G_{\mu}^{(3)}\right)_{k_{1} k_{2}}$ field. The difference has to do with breakdown of parity in the weak interactions.

By using the expansion (2.9) one can write the chiral components for an $S U(2)$ rank 2 spinor, defined in (2.4)-(2.7), in terms of the usual vector and tensor fields as:

$$
\begin{aligned}
& \psi_{R R i_{1} i_{2}, a_{1} a_{2}}^{(2)}=\left(\frac{\left(1+\gamma^{5}\right)}{2} \sigma^{\mu v} C\right)_{a_{1} a_{2}}\left(\mathcal{F}_{\mu \nu}\right)_{i_{1} i_{2}}+\left(\frac{\left(1+\gamma_{5}\right)}{2} C\right)_{a_{1} a_{2}}\left(\phi+\phi^{A}\right)_{i_{1} i_{2}} \\
& \psi_{L L i_{1} i_{2}, a_{1} a_{2}}^{(2)}=\left(\frac{\left(1-\gamma^{5}\right)}{2} \sigma^{\mu v} C\right)_{a_{1} a_{2}}\left(\mathcal{F}_{\mu \nu}\right)_{i_{1} i_{2}}+\left(\frac{\left(1-\gamma_{5}\right)}{2} C\right)_{a_{1} a_{2}}\left(\phi-\phi^{A}\right)_{i_{1} i_{2}} \\
& \psi_{R L i_{1} i_{2}, a_{1} a_{2}}^{(2)}=\left(\frac{\left(1+\gamma^{5}\right)}{2} \gamma^{\mu} C\right)_{a_{1} a_{2}}\left(G_{\mu}^{V}+G_{\mu}^{A}\right)_{i_{1} i_{2}} \\
& \psi_{L R i_{1} i_{2}, a_{1} a_{2}}^{(2)}=\left(\frac{\left(1-\gamma^{5}\right)}{2} \gamma^{\mu} C\right)_{a_{1} a_{2}}\left(G_{\mu}^{V}-G_{\mu}^{A}\right)_{i_{1} i_{2}} .
\end{aligned}
$$

In our approach all tensor fields are independent variables. The equations of motion satisfied by the chiral components leads to the usual relations between $\mathcal{F}_{\mu \nu}$ and derivatives of vector fields and, for internal 
symmetries, the fields themselves [7-9]. In this sense, gauge invariance can be derived [8,9]. The scalar fields give rise to gauge fixing terms in the Lagrangian [10].

One decomposes the fields $G_{\mu}^{V}$ and $G_{\mu}^{A}$ in terms of singlet fields $\left(a_{\mu}^{(0)}\right.$ and $\left.a_{5 \mu}^{(0)}\right)$ and triplet fields $\left(a_{\mu}^{(\ell)}\right.$ and $\left.a_{5 \mu}^{(\ell)}\right)$ by using the basis formed by the Pauli matrices $\sigma^{(\ell)}(\ell=1,2,3)$ and the unit matrix as:

$$
\begin{aligned}
& \left(G_{\mu}^{V}\right)_{i_{1} i_{2}}=\frac{1}{4}\left(a_{\mu}^{(0)} \delta_{i_{1} i_{2}}-a_{\mu}^{(\ell)} \sigma_{i_{1} i_{2}}^{(\ell)}\right) \\
& \left(G_{\mu}^{A}\right)_{i_{1} i_{2}}=\frac{1}{4}\left(a_{5 \mu}^{(0)} \delta_{i_{1} i_{2}}-a_{5 \mu}^{(\ell)} \sigma_{i_{1} i_{2}}^{(\ell)}\right)
\end{aligned}
$$

In view of its relevance, from the point of view of the approach here proposed, let us consider the sum over the chiral components $\psi_{R L}^{(2)}$ and $\psi_{L R}^{(2)}$ :

$$
\left(\psi_{R L}^{(2)}+\psi_{L R}^{(2)}\right)_{i_{1} i_{2}}=\gamma^{\mu}\left(G_{\mu, i_{1} i_{2}}^{V}-\gamma^{5} G_{\mu, i_{1} i_{2}}^{A}\right) C \equiv \gamma^{\mu}\left(G_{\mu}\right)_{i_{1} i_{2}} C .
$$

The above expression is a parity conserving combination of spinor components.

By using the usual expressions for the Pauli $\sigma$ matrix one writes the vector and axial vector components $\left(G_{\mu}^{V}\right.$ and $\left.G_{\mu}^{A}\right)$ given in (2.14), as the matrices:

$$
\begin{aligned}
& G_{\mu}^{V}=\frac{1}{4}\left(\begin{array}{cc}
a_{\mu}^{(0)}-a_{\mu}^{(3)} & -a_{\mu}^{(1)}+i a_{\mu}^{(2)} \\
-a_{\mu}^{(1)}-i a_{\mu}^{(2)} & a_{\mu}^{(0)}+a_{\mu}^{(3)}
\end{array}\right) \\
& G_{\mu}^{A}=\frac{1}{4}\left(\begin{array}{cc}
a_{5 \mu}^{(0)}-a_{5 \mu}^{(3)} & -a_{5 \mu}^{(1)}+i a_{5 \mu}^{(2)} \\
-a_{5 \mu}^{(1)}-i a_{5 \mu}^{(2)} & a_{5 \mu}^{(0)}+a_{5 \mu}^{(3)}
\end{array}\right) .
\end{aligned}
$$

In the next section we shall use the axial component $G_{\mu}^{A}(2.17)$ in order to develop a theory in which parity is broken.

\section{INTERACTIONS}

In this section we shall discuss how an unified theory can be formulated by using spinor fields in the description of bosons and fermions.

The most general Lagrangian involving the interaction of fields can be written as

$$
\mathcal{L}=\mathcal{L}_{0}+\mathcal{L}_{I}
$$

where $\mathcal{L}_{0}$ is the kinetic term of the Lagrangian. This term contains only space-time derivatives of fields. We shall see that by using spinors the kinetic term involves only first order space-time derivatives of the dynamical variables. $\mathcal{L}_{I}$ in is the interaction Lagrangian.

By assuming left-handed neutrinos, we write the kinetic Lagrangian for fermions as:

$$
\mathcal{L}_{0}^{(F)}=\bar{L}^{(F)}(i \not \partial) L^{(F)}
$$


Whereas the kinetic Lagrangian for bosons, written in terms of the chiral components, is given by:

$$
\mathcal{L}_{0}^{(B)}=\bar{\psi}_{R R} \cdot\left(i \not \partial \psi_{R L}\right)+\bar{\psi}_{R L} \cdot\left(i \not \partial \psi_{L L}\right)+\bar{\psi}_{L R} \cdot\left(i \not \partial \psi_{R R}\right)+\bar{\psi}_{L L} \cdot\left(i \not \partial \psi_{L R}\right)
$$

The Interaction Lagrangian can always be written as a sum involving powers of fields

$$
\mathcal{L}_{I}=\mathcal{L}_{I}^{2}+\mathcal{L}_{I}^{3}+\mathcal{L}_{I}^{4}
$$

The term involving quadratic interactions of fields is $\mathcal{L}_{I}^{2}$. The cubic interactions are described by the $\mathcal{L}_{I}^{3}$ term and, if one has scalar fields, the term $\mathcal{L}_{I}^{4}$ contains quartic interactions. Renormalizability of the theory restricts the number of possible couplings.

The most general interaction Lagrangian is a functional of the dynamical variables under the general form

$$
\mathcal{L}_{I}=\mathcal{L}_{I}\left[L_{i}^{(F)}, q_{i, k}^{(F)}, \psi_{L R, i_{1} i_{2}}^{(2)} \ldots \psi_{L L, i_{1} i_{2}}^{(2)}, \psi_{L R, k_{1} k_{2}}^{(3)} \ldots \psi_{L L, k_{1} k_{2}}^{(3)}, \varphi\right]
$$

where $\varphi$, in (3.5), represents scalar fields.

When dealing with rank 2 spinors some of the quadratic interaction terms involves masses as coefficients of product of fields. One can provide masses to the $W_{\mu}^{(+)}, W_{\mu}, Z_{\mu}$ particles through the use of the Higgs mechanism or without invoking the Higgs mechanism. Masses will be discussed in a future publication. We shall assume here that the photon is massless and that $W_{\mu}^{(+)}, W_{\mu}, Z_{\mu}$ are massive. We do not assume, in this paper, a specific mechanism for providing masses to particles.

The most general, renormalizable and invariant Lagrangian describing the interaction of fermions with the intermediate bosons is:

$$
\mathcal{L}_{I}^{\text {fermions }}=g \bar{L}^{(F)}\left(\psi_{R L}^{(2)}+\psi_{L R}^{(2)}\right)\left(C^{-1}\right) L^{(F)}
$$

where $g$ is the electroweak $S U(2)$ coupling constant. By writing the Lagrangian interaction in terms of the fields $L^{(F)}$ we break parity. This is due to the fact that, from expression (2.1), we are coupling, in Lagrangian (3.6), chiral components of some leptonic fields.

By using expressions (2.12) and (2.13) for the $\psi_{R L}$ and $\psi_{L R}$ fields we write the fermionic Lagrangian defined in (3.6) as:

$$
\mathcal{L}_{I}^{\text {fermions }}=g \bar{L}^{(F)} \gamma^{\mu}\left(G_{\mu}^{V}-\gamma^{5} G_{\mu}^{A}\right) L^{(F)}
$$

The above electroweak Lagrangian is $S U(2)$ symmetric. In the next section we will break the $S U(2)$ symmetry by expressing a sum of singlet fields and the third component of triplet fields as mixings of the neutral electroweak fields $A_{\mu}$ and $Z_{\mu}$. This is very similar (but not equal) to Weinberg's mixings. The way we break parity is, however, completely different.

Within the $V-A[16,17]$ theory the coupled currents have a vector and an axial component. In our approach we have a two component theory at the level of the dynamic variables associated to the intermediate bosons. 


\section{BREAKDOWN OF SPACE-REFLECTION AND $S U(2)$ SYMMETRIES}

We shall discuss in this section some aspects of discrete symmetries [12-14]. For this reason we shall start this section by introducing the transformation properties of rank 2 spinor fields under discrete symmetries.

Under space reflection $(\vec{x} \rightarrow-\vec{x})$ a rank 1 spinor field transforms as

$$
\psi_{a_{1}}(\vec{x}, t) \rightarrow \lambda_{P} \gamma_{a_{1} a_{1}^{\prime}}^{0} \psi_{a_{1}^{\prime}}(-\vec{x}, t)
$$

whereas a rank 2 spinor field transforms as

$$
\psi_{a_{1} a_{2}}(\vec{x}, t) \rightarrow \lambda_{P} \gamma_{a_{1} a_{1}^{\prime}}^{0} \gamma_{a_{2} a_{2}^{\prime}}^{0} \psi_{a_{1}^{\prime} a_{2}^{\prime}}(-\vec{x}, t)
$$

Under $C P T$ a rank 1 spinor field transforms as

$$
\psi_{a_{2}}(x) \rightarrow \lambda_{C P T} \gamma_{a_{1} a_{1}^{\prime}}^{5} \psi_{a_{1}^{\prime}}(-x),
$$

whereas a rank 2 spinor field transforms, under $C P T$, as

$$
\psi_{a_{1} a_{2}}(x) \rightarrow \lambda_{C P T} \gamma_{a_{1} a_{1}^{\prime}}^{5} \gamma_{a_{2} a_{2}^{\prime}}^{5} \psi_{a_{1}^{\prime} a_{2}^{\prime}}(-x)
$$

The extension from the rank 1 to rank 2 spinor fields for other symmetries is straightforward [15].

One of the distinctive features of the weak interactions is that space-reflection symmetry is broken by these interactions. The possible existence of just one left-handed neutrino seems to emphasize this aspect.

In the Glashow-Weinberg-Salam (G.W.S.) model $[1,2]$ some of the dynamical variables of the theory are left and right-handed components of fermionic fields. Left and right components belongs in this formulation to different representations of the $S U(2)$ group. A differentiation between left and right lies in the very foundations of the theory. This asymmetric treatment of chiral components allows for the incorporation of parity violation in the theory.

Another relevant feature, from the point of view of symmetries, is that an internal - SU(2) symmetry - is also broken by these interactions. In order to break $S U(2)$ symmetry usually one makes use of two mechanisms.

The first mechanism is the spontaneous breakdown of gauge symmetry [3]. The particles associated to the $Z_{\mu}, W_{\mu}^{+}, W_{\mu}$ fields can acquire mass through the Higgs mechanism [18-22].

The second mechanism is the electroweak mixing of fields [3]. The electroweak mixings break $S U$ (2) symmetry in such a way as to introduce electromagnetic interaction of leptons. It is, in this way, a mechanism to assign electric charges to leptons.

In this paper we pursue further the idea that breakdown of parity is an intrinsic property of the weak interactions and that this should be described through the choice of variables. Since space-reflection asymmetry is manifested in phenomena involving the interactions of the intermediate vector bosons we prefer, however, to use parity asymmetric boson variables. As we shall see in this section, this can be done by using rank 2 spinors.

A rank 2 spinor field has enough degrees of freedom to make possible the breakdown of parity and $S U$ (2) symmetries at the level of the bosonic variables. $S U(2)$ symmetry and space-reflection will become, in this way, broken symmetries. 
Let us analyse first the breakdown of parity. We shall use the axial component fields $a_{5 \mu}^{(0)}, a_{5 \mu}^{(1)}$ and $a_{5 \mu}^{(2)}$ in order to build a parity breaking theory. We break parity in the charged sector by choosing:

$$
a_{5 \mu}^{(1)}=a_{\mu}^{(1)}, \quad a_{5 \mu}^{(2)}=a_{\mu}^{(2)} .
$$

The above choices are needed in order to reproduce the $V$ - $A$ theory of the weak interactions [16-17]. In order to break parity in the neutral sector we choose:

$$
a_{5 \mu}^{(0)}=\sqrt{1+\xi^{2}} Z_{\mu}
$$

where $\xi$ is a fundamental parameter of the electroweak model $\left(\xi=\tan \theta_{w}\right)$. This is the only free parameter to be introduced in our model. This parameter is an asymmetry parameter. It gives a measure of an asymmetry between the neutral and charged sectors resulting from different parity breaking interaction strengths. For $\xi=1$ the neutral and charged sectors break parity with equal strengths. We shall see that for $\xi=0$ the $S U(2)$ symmetry is restored.

From the above expressions one can see that space-reflection asymmetry becomes an asymmetry which is dealt with at the level of the dynamic variables of the intermediate bosons. This brings breakdown of parity to the realm of the weak interactions.

One can easily check that by substituting pseudovector fields by vector fields one is led to a theory in which parity is broken. By means of the choices (4.5) and (4.6) one is making an explicit breakdown of parity.

We notice that up to this point we have broken only space-reflection symmetry. By using the most general rank 2 spinor, one can have two components each of them $S U(2)$ symmetric. The two singlets associated to the components are the $a_{\mu}^{(0)}$ and the redefined $a_{5 \mu}^{(0)}$ fields.

One breaks global $S U(2)$ symmetry by introducing the following $\xi$ dependent electroweak mixings:

$$
\begin{aligned}
& a_{\mu}^{(0)}+a_{5 \mu}^{(0)}=\frac{2 \xi}{\sqrt{1+\xi^{2}}}\left(A_{\mu}-\xi Z_{\mu}\right) \\
& a_{\mu}^{(3)}+a_{5 \mu}^{(3)}=\frac{2}{\sqrt{1+\xi^{2}}}\left(\xi A_{\mu}+Z_{\mu}\right)
\end{aligned}
$$

One writes the $W_{\mu}$ and $W_{\mu}^{+}$fields as the linear combinations:

$$
W_{\mu}=\frac{1}{\sqrt{2}}\left\{a_{\mu}^{(1)}+i a_{\mu}^{(2)}\right\}, \quad W_{\mu}^{+}=\frac{1}{\sqrt{2}}\left\{a_{\mu}^{(1)}-i a_{\mu}^{(2)}\right\} .
$$

By taking $\xi=\tan \theta_{w}$, one can see, from (4.7) and (4.8), that the choice:

$$
a_{5 \mu}^{(3)}=0,
$$

permit us to identify our vector triplet fields with the triplet fields of Weinberg [3].

It follows from the first definition in eq. (4.7), that the sum over the singlet fields introduced by us play the role of the $U(1)$ vector field of the GWS theory [1-3]. That is,

$$
a_{\mu}^{(0)}+a_{5 \mu}^{(0)}=2 \xi B_{\mu} .
$$


For the choices (4.5)-(4.9) we get the following expressions for the $G_{\mu}^{V}$ and $G_{\mu}^{A}$ matrices, defined in (2.16) and (2.17):

$$
\begin{gathered}
G_{\mu}^{V}=\left(\begin{array}{cc}
0 & 0 \\
0 & \frac{1}{\sqrt{1+\xi^{2}}}\left(\xi A_{\mu}+Z_{\mu}\right)
\end{array}\right)+\left(\begin{array}{cc}
-\frac{3}{4} \sqrt{1+\xi^{2}} Z_{\mu} & -\frac{1}{2 \sqrt{2}} W_{\mu}^{+} \\
-\frac{1}{2 \sqrt{2}} W_{\mu} & -\frac{3}{4} \sqrt{1+\xi^{2}} Z_{\mu}
\end{array}\right) \\
G_{\mu}^{A}=\left(\begin{array}{cc}
\frac{1}{4} \sqrt{1+\xi^{2}} Z_{\mu} & -\frac{1}{2 \sqrt{2}} W_{\mu}^{+} \\
-\frac{1}{2 \sqrt{2}} W_{\mu} & \frac{1}{4} \sqrt{1+\xi^{2}} Z_{\mu}
\end{array}\right)
\end{gathered}
$$

The conclusion is that we can use the $G_{\mu}^{V}$ and $G_{\mu}^{A}$ components of a rank 2 spinor $\psi$ in order to get a set of dynamical variables that incorporates the breakdown of symmetries at the level of bosonic variables.

Besides incorporating both asymmetries through the choice of the bosonic field variables, we break these symmetries in the same way. In both cases the symmetries are broken by imposing that certain fields $\left(a_{5 \mu}^{(0)}, a_{5 \mu}^{(1)}, a_{5 \mu}^{(2)}\right.$ and $\left.a_{\mu}^{(3)}\right)$ transforms in a way that is different from the way we would expect if the symmetries were realized in nature.

In this section we have shown how one can use both components $\left(G_{\mu}^{V}\right.$ and $\left.G_{\mu}^{A}\right)$ of a rank 2 spinor as $S U(2)$ and space-reflection broken dynamical variables describing the intermediate bosons. The asymmetries are reflected in the lack of symmetries of these bosonic variables.

\section{COUPLING CONSTANTS AND ASYMMETRIES}

By substituting expressions (4.11)-(4.12) in (3.7), we get the electroweak interaction Lagrangian for fermions belonging to the F-family. For the first family this Lagrangian is:

$$
\begin{aligned}
\mathcal{L}^{\text {electroweak }}= & g\left(\bar{v}_{e L}, \bar{e}\right) \gamma^{\mu}\left\{\left(\begin{array}{cc}
-\sqrt{1+\xi^{2}} Z_{\mu} & 0 \\
0 & \frac{\xi A_{\mu}}{\sqrt{1+\xi^{2}}}-\frac{\xi^{2}}{\sqrt{1+\xi^{2}}} Z_{\mu}
\end{array}\right)\right. \\
& \left.+\frac{1-\gamma^{5}}{4}\left(\begin{array}{cc}
-\sqrt{1+\xi^{2}} Z_{\mu} & -\sqrt{2} W_{\mu}^{+} \\
-\sqrt{2} W_{\mu} & +\sqrt{1+\xi^{2}} Z_{\mu}
\end{array}\right)\right\}\left(\begin{array}{c}
v_{e L} \\
e
\end{array}\right)
\end{aligned}
$$

Where $e$ is the electron field and $v_{e L}$ is the left-handed neutrino field.

The electroweak interactions involves, from (V.1), 4 different coupling constants. They depend on the 
parameter $\xi$ as follows:

$$
\begin{aligned}
& g \frac{1}{\sqrt{1+\xi^{2}}} \xi \\
& g \frac{1}{\sqrt{1+\xi^{2}}} \xi^{2} \\
& g \frac{1}{4} \sqrt{1+\xi^{2}} \\
& g \frac{1}{4} \sqrt{2}
\end{aligned}
$$

The first $\xi$-dependent parameter is the electric charge of the positron. As can be seen from (V.1), the electromagnetic interaction of leptons is, in our approach, a consequence of the lack of $S U$ (2) symmetry of the fundamental interactions.

Let us analyse some specific values of the parameter $\xi$. Let us consider first the value of this parameter for which one gets a symmetry between the charged and the neutral sectors in the parity broken couplings. As pointed out before, this situation occurs when $\xi=1$. For this value of $\xi$ the theory contains only two coupling constants. One coupling for the parity broken piece of the lagrangian and another coupling for the parity preserving term of the lagrangian. They differ by just a factor of 2 .

We shall analyse now the aspect of space reflection symmetry. A theory is left-right asymmetric if the theory is not invariant under the exchange of left components by rigth components $(R \leftrightarrow L)$.

We define a theory as being completely left-right asymmetric (under the exchange of $R \leftrightarrow L$ ) if only left-handed components are coupled. Under these circumstances the Lagrangian is term by term left-right asymmetric. One can define a theory as maximally allowed parity broken if the electromagnetic interaction is the only parity preserving interaction of the Lagrangian. This differentiation is needed in view of the fact that electromagnetic interactions are parity conserving.

We analyse now the conditions under which leptonic interactions (for left-handed neutrinos) become completely space-reflection asymmetric. A completely broken parity symmetry occurs when $\xi=0$. For this value of the $\xi$ parameter the lagrangian (5.1) becomes:

$$
\mathcal{L}^{\text {electroweak }}=g \bar{\ell}_{L}\left(\begin{array}{cc}
-\frac{1}{2} Z_{\mu} & -\frac{1}{\sqrt{2}} W_{\mu} \\
-\frac{1}{\sqrt{2}} W_{\mu}^{+} & \frac{1}{2} Z_{\mu}
\end{array}\right) \ell_{L}+g \bar{q}_{L}\left(\begin{array}{cc}
-\frac{1}{2} Z_{\mu} & -\frac{1}{\sqrt{2}} W_{\mu} \\
-\frac{1}{\sqrt{2}} W_{\mu}^{+} & \frac{1}{2} Z_{\mu}
\end{array}\right) q_{L}
$$

where $q_{L}$ and $\ell_{L}$ are the all left-handed components of the $q$ and $\ell$ variables.

As we can see from (5.6) the $\xi=0$ limit leads us to a $S U(2)$ symmetric model in which we turn off electrodynamics and the $Z_{\mu}$ field plays the role of the third component of the triplet field. In this limit parity is totally broken. We get, in this limit a model quite similar to unbroken $S U(2)$ model of GWS model.

The conclusion is that a theory for which parity is completely broken, is a theory for which $S U(2)$ symmetry is restored. This is possible only when we get rid of the electromagnetic interactions. 
Let us consider finally the conditions under which electromagnetic interactions is the only interaction preserving parity. In order to do so we separate out the electromagnetic interaction from the weak interactions. We write

$$
\begin{aligned}
\mathcal{L}^{\text {electroweak }}= & -g_{e} \bar{e} \gamma^{\mu} e A_{\mu}+g_{w}\left(\bar{v}_{e L}, \bar{e}\right) \gamma^{\mu}\left\{\begin{array}{cc}
-\frac{1}{\cos \theta_{w}} Z_{\mu} & 0 \\
0 & -\frac{\sin ^{2} \theta_{w}}{\cos \theta_{w}} Z_{\mu}
\end{array}\right) \\
& \left.+\left(1-\gamma_{5}\right)\left(\begin{array}{cc}
\frac{1}{4 \cos \theta_{w}} Z_{\mu} & -\frac{1}{2 \sqrt{2}} W_{\mu} \\
-\frac{1}{2 \sqrt{2}} W_{\mu}^{+} & \frac{1}{4 \cos \theta_{w}} Z_{\mu}
\end{array}\right)\right\}\left(\begin{array}{l}
v_{e L} \\
e
\end{array}\right) .
\end{aligned}
$$

Where in the last equation we have written the $\xi$ parameter as

$$
\xi=\tan \theta_{w}
$$

The last four terms of the Lagrangian (5.7) of the electroweak interactions couples only left-handed components of electrons and neutrinos. In order to analyse under which circunstances there are no parity conserving terms in the weak interactions of leptons one needs to consider only the weak interaction of electrons with the neutral boson $Z_{\mu}$.

The choice

$$
\sin ^{2} \theta_{w}=\frac{1}{4}
$$

leads us, for left-handed neutrinos, to a Lagrangian of the weak interactions that can be written in terms of chiral components, as:

$$
\begin{aligned}
\mathcal{L}^{\text {weak }}= & \frac{g_{w}}{4 \cos \theta_{w}}\left(\overline{e_{L}} \gamma^{\mu} Z_{\mu} e_{L}-\overline{e_{R}} \gamma^{\mu} Z_{\mu} e_{R}-2 \overline{\nu_{e L}} \gamma^{\mu} Z_{\mu} v_{e L}\right) \\
& -\frac{g_{w}}{\sqrt{2}}\left(\overline{v_{e L}} \gamma^{\mu} W_{\mu}^{+} e_{L}+\overline{e_{L}} \gamma^{\mu} W_{\mu}^{-} v_{e L}\right) .
\end{aligned}
$$

One can easily check that for the value (5.9) of Weinberg's $\theta$-angle the weak interaction of leptons is maximally allowed left-right asymmetric. An example of a simple gauge group for the electroweak interactions for which $\sin ^{2} \theta_{w}=\frac{1}{4}$ is given in ref. (23).

If maximally allowed asymmetry between left and right in the weak interaction is a requirement to be satisfied by the weak interaction, then the $\theta_{w}$ parameter would no longer be a free parameter. It would be determined from symmetry arguments. The fact that the experimental value of $\theta_{w}$ is so close to (5.9) means that the weak interactions is very close to being left-right asymmetric.

\section{CONCLUSIONS}

In this paper we have presented an unified theory of the fundamental interactions by using spinor fields [5]. The use of spinors brings to the particle description a new set of tools. No use is made, when using spinors, 
of gauge invariance. The dynamical variables, in the spinorial approach, is a set of $2^{2 s}$ chiral components for each spin $s$ particle.

The use of a rank 2 spinor fields allows us to built explicit space reflection asymmetric theories. This is done, formally, by interchanging pseudovector fields with vector fields. The use of spinors allows us to see that the electroweak interactions are intrinsically asymmetric with regard to space reflection and that this asymmetry can be dealt with at the level of the variables associated to the intermediate bosons.

The theory of the fundamental interactions deals with coupling constants and other fundamental parameters, like masses, whose understanding is a great scientific challenge today. As an application of our approach, we have discussed in this paper how the coupling constants of the electroweak interactions are related to breakdown of symmetries.

In the electroweak interactions we deal with 4 coupling constants. These couplings arises in the process of breaking $S U(2) \times U(1)$ symmetry as suggested by the Standard Model, or as a result of parity and $S U(2)$ symmetries as proposed here. There are great conceptual differences between our approach and the one proposed by Glashow, Weinberg and Salam. In both theories the 4 couplings depend on just one parameter. In the Standard Model this parameter is the $\theta_{w}$ parameter whereas in our approach this parameter is the $\xi$ parameter.

In our approach there is an interplay between breakdown of parity and $S U(2)$ symmetries. This interplay is reflected in the values of the four coupling constants. When we recover $S U(2)$ symmetry, in the $\xi=0$ limit, we loose parity in a complete way. This happens in the limit in which the electric charges of all fermions are zero.

We have also suggested that symmetry arguments can be evoked in such a way as to guess a value for the asymetry parameter that is very close to the experimentally observed value of this parameter. We suggest also that the value of $\sin ^{2} \theta_{w}$ is expected, from parity asymmetry arguments, to assume the value $\frac{1}{4}$. At this value the usual weak interactions becomes a parity breaking interaction with no parity preserving terms in the Lagrangian. The theory of the electroweak interactions is close to a maximally allowed parity violating theory. From this argument it follows that $g_{w}$ should be, from (5.7), equal to $2 g_{e}$. An example of a simple gauge group for the electroweak interactions for which $\sin ^{2} \theta_{w}=\frac{1}{4}$ is given in ref. (23).

The fact that quarks and leptons have different charges can be explained on the other hand as a consequence of an extra symmetry ( $S U(3))$ of the interactions of quarks (Marques and Spehler 2007, unpublished data).

The conclusion is that symmetries can be evoked in order to give an account for relations among coupling constants and for the values of the electric charges of fermions.

\section{ACKNOWLEDGMENTS}

The authors would like to acknowledge fruitful discussions with Prof. S.F. Novaes and O.J.P. Éboli. One of the authors (D.S.) would like to thank the members of the GRAFITE group for their hospitality and support. One of us (G.C.M.) would like the acknowledge the support of the Fundação de Amparo à Pesquisa do Estado de São Paulo (FAPESP). 


\section{RESUMO}

Com base em uma formulação nova para simetrias das interações fundamentais nós lidamos, neste trabalho, com interações eletrofracas de leptons. Mostramos que as constantes do acoplamento, associadas aos acoplamentos de bósons intermediários, podem ser entendidas como parâmetros associados à quebra de simetrias SU (2) e paridade. A quebra de ambas as simetrias é caracterizada por um parâmetro novo (o parâmetro de assimetria) das interações eletrofracas. Este parâmetro dá uma medida da intensidade com que a simetria é quebrada. Analisamos o comportamento da teoria para três valores deste parâmetro. O valor mais relevante é aquele para o qual apenas as interações eletromagnéticas não quebram a paridade (a teoria assimétrica esquerda-direita permitida da maneira máxima). A assimetria máxima permitida é uma exigência que leva a um ângulo de Weinberg cujo valor é próximo daquele observado experimentalmente.

Palavras-chave: simetrias, constantes do acoplamento, interações eletrofracas.

\section{REFERENCES}

[1] GLASHOW SL. 1961. Partial-symmetry of weak interactions. Nucl Phys 22: 579.

[2] Salam A. 1968. In: Elementary Particle Physics (Nobel Symposium no. 8). Edited by N. Svartholm (Almqvist and Wiksell, Stockholm), p. 367.

[3] Weinberg S. 1967. A Model of leptons. Phys Rev Lett 19: 1264-1266.

[4] YANG CN AND Mills RL. 1954. Conservation of isotopic spin and isotopic gauge invariance. Phys Rev 96: $191-195$

[5] Bargmann AND Wigner E. 1948. Group theoretical discussion of relativistic wave equations. Proc Natl Acad Sci. 34: 211-223.

[6] Marques GC AND SPEHLER D. 1996. Left right asymmetry and minimal coupling. J Math Phys. 37: 174-195.

[7] Marques GC And Spehler D. 1999. Chirality in electrodynamics. Int J Mod Phys A 14: 5121-5135.

[8] Marques GC And Spehler D. 2001. Chirality in the context of spin 3/2 particles. J Math Phys. 42: 1599-1611.

[9] Marques GC And Spehler D. 1999. Chiral asymmetry and gauge invariance. Mod Phys Lett A. 14: 13171327.

[10] Marques GC And Spehler D. 2003. Magnetic monopoles and chiral asymmetry. Int J Mod Phys A. 18: 2457-2475.

[11] SPEHLER D AND MARQUES GC. 2005. Unified electroweak model based on space-time and isospin symmetries. Int J Mod Phys A. 20: 175-198.

[12] Muirhead H. 1968. The physics of elementary particles. Pergamon Press.

[13] Mohapatra RN. 1991. Massive neutrinos in physics and astrophysics. Singapore New Jersey, World Scientific.

[14] Abers ES And LeE BW. 1973. Gauge theories. Phys Rep. 9C: 1-141.

[15] Weinberg S. 1996. The quantum theory of fields, Cambridge University Press. 
[16] Feynman RP and Gell-Mann M. 1958. Theory of the Fermi interaction. Phys Rev 109: 193-198.

[17] Sudarshan ECG AND Marshak RE. 1958. Chirality invariance and the universal Fermi interaction. Phys Rev 109: 1860-1862.

[18] Higgs PW. 1964. Large angle p-p elastic scattering at 30 bev. Phys Rev Lett 12: 132-133.

[19] Higgs PW. 1964. Broken symmetries and the masses of gauge bosons. Phys Rev Lett 13: 508-509.

[20] Higgs PW. 1966. Spontaneous symmetry breakdown without massless bosons. Phys Rev 145: 1156-1163.

[21] Guralnik GS, Hagen CR And KibBle TWB. 1964. Global conservation laws and massless particles. Phys Rev Lett 13: 585-587.

[22] ENGLERT F AND BRout R. 1964. Broken symmetry and the mass of gauge vector mesons. Phys Rev Lett 13: $321-323$.

[23] WeInberg S. 1972. Effects of a neutral intermediate boson in semileptonic processes. Phys Rev D 5: 14121417. 